Voix et Images

\title{
Revenue des utopies : Nicole Brossard devant l'histoire contemporaine
}

\section{Patricia Smart}

Volume 13, numéro 3 (39), printemps 1988

Jack Kerouac et l’imaginaire québécois

URI : https://id.erudit.org/iderudit/200740ar

DOI : https://doi.org/10.7202/200740ar

Aller au sommaire du numéro

Éditeur(s)

Université du Québec à Montréal

ISSN

0318-9201 (imprimé)

1705-933X (numérique)

Découvrir la revue

Citer cet article

Smart, P. (1988). Revenue des utopies : Nicole Brossard devant l'histoire

contemporaine. Voix et Images, 13(3), 496-499. https://doi.org/10.7202/200740ar d'utilisation que vous pouvez consulter en ligne.

https://apropos.erudit.org/fr/usagers/politique-dutilisation/ 


\section{Revenue des utopies: Nicole Brossard devant l'histoire contemporaine}

\section{par Patricia Smart, Université Carleton}

Observant, depuis quelque temps, la spirale aérienne du trajet de Nicole Brossard, j'ai plus d'une fois pensé que, selon la logique même de sa course, elle devait se trouver sur une spire qui allait bientôt la rapprocher de la terre. Eh bien, c'est ce qui s'est produit avec son dernier roman, et on ne peut qu'espérer qu'elle décidera d'y séjourner quelque peu encore. Le Désert mauve ${ }^{1}$ est sûrement le plus beau livre de sa carrière - un grand roman courageux et lucide, qui traverse tout le désespoir de notre civilisation et qui, grâce à la générosité de sa créatrice, nous ouvre un chemin vers l'espérance.

Quelques allusions passagères à un «Dante's View Motel» dans ce fabuleux «roman du désert» font comprendre qu'il s'agit bel et bien de la Divine (et humaine) Comédie d'une auteure qui se sait rendue au mi-temps de sa vie. Revenue des utopies (sans avoir abandonné pour autant son désir de transformer le monde), Brossard trouve dans le désert de l'Arizona une métaphore de la totalité de l'expérience humaine. Dans cet espace où tout se réduit à l'essentiel, elle se livre, par des mots d'une poésie et d'une précision étonnantes, à la recherche d'un sens capable de racheter l'humanité dans la nuit de cette fin de siècle. Le désert est indescriptible (p. 11): ainsi commence ce roman mystique et très incarné, toutés perspectives ouvertes sur l'espace «hyperréel» du désert - à la fois lieu de violence (expérience scientifique et explosion imminente), lieu post-moderne parsemé de traces humaines (canettes et carrosseries d'autos, motels anonymes où l'on vit autour de la piscine et dans les bars) et lieu de beauté indicible faite de lumière, de couleurs, d'une végétation lente et patiente qui sait résister à la mort pendant de longues périodes d'aridité. Lieu qui peut enseigner la force, si l'on sait confronter sa nuit et en revenir: L'humanité était fragile parce qu'elle ne 
soupçonnait pas l'existence de l'Arizona. So fragile (p. 13). Pour l'apprentissage du désert, il faut un guide, et c'est le rôle que joue Brossard pour ses lecteurs et lectrices.

De la nuit à la possibilité de l'aube, grâce à toute une série d'échanges entre femmes, une traversée se fait, reflétée dans la modification que subit le roman à l'intérieur du roman (le Désert mauve de l'Américaine Laure Angstelle) à mesure que se prépare sa version traduite, qui est elle-même contenue dans le texte (Mauve, L'Horizon, traduit par la Québécoise Maude Laures). Tout le sens du roman tient dans le déplacement de la première version à la deuxième, dans la rencontre entre une auteure et sa traductrice, grâce à la lecture et à la réécriture du texte original. Mais le passage ne se fait ni sans ambiguités, ni sans perte, puisque chacune des deux versions de l'intrigue aboutit à un événement apparemment incontournable - le meurtre de la femme «intégrale» vers laquelle se dirigeaient tout le désir et tout le sens de la possibilité du roman de Laure Angstelle: Le ravage est grand (p. 220).

Un des plaisirs de la lecture de ce roman est d'assister au tour de force par lequel Brossard nous présente, en français, non seulement le texte original du roman, mais aussi sa traduction. Identiques jusque dans leur pagination et leur division en phrases et en paragraphes, les deux versions sont pourtant l'expression de deux sensibilités différentes, et c'est dans la variation de mots et d'images de la première à la deuxième qu'émerge le mouvement vers une réconciliation avec le temps et le réel. Même prise isolément, la première version (avec sa préoccupation constante de «la réalité») représenterait un point tournant important dans l'évolution de l'écriture de Brossard. Mais c'est en faisant passer ce premier récit tout imprégné d'intensité, de vitesse et de violence à travers l'intelligence amoureuse et toute en simplicité de la traductrice Maude Laures que Brossard parvient à enraciner sa vision du désert et à l'insérer dans la durée. De Laure Angstelle, on ne sait rien, sauf qu'elle est l'auteure d'un roman plein de passion, d'intensité, d'amour de l'écriture. De Maude Laures, la narratrice ou protagoniste d'une longue partie charnière ("Un livre à traduire») qui précède la version traduite, on apprend qu'elle enseigne dans un collège de filles, qu'elle joue au tennis, que depuis qu'elle porte des lentilles, elle ne se maquille plus les yeux, qu'elle aime voyager en auto entre Montréal et Québec, que chaque jour de paye, elle achète cinq ou six livres dont elle savait que la lecture allait la rassurer sur l'existence ou conforter quelque part au fond d'elle-même les aléas de ses pensées (p. 122). Bref, elle est «réelle», elle a le goût de vivre, et les réflexions qu'elle couche sur le papier concernant le roman qui l'obsède («Lieux et objets», «Personnages», «Scènes», «Dimensions») font vivre ce roman de nouveau, et autrement, que dans le texte original.

Que contient donc le court roman de Laure Angstelle qui bouleverse à ce point la vie de Maude Laures? Narré par une adolescente de quinze ans, Mélanie (Mais la nuit, lui dit sa mère), il raconte les fugues nocturnes de celle-ci dans le désert, dans la Météor de sa mère Kathy Kérouac, propriétaire du Red Arrow Motel, ses retours à la routine diurne autour de la piscine du motel, ses rapports 
ambivalents avec sa mère et avec l'amante de celle-ci, Lorna, et surtout la découverte foudroyante d'une femme, Angela Parkins, géomètre âgée de 40 ans qui travaille avec un groupe d'hommes à un projet mystérieux et hautement technologique dans le désert, et qui, en compagnie de ses compagnons de travail, visite le bar du motel une fois par mois. En outre, chaque chapitre du récit de Mélanie débute par une page présentée par un narrateur omniscient et racontant une histoire parallèle à celle de l'adolescente: l'arrivée d'un personnage identifié comme «l'homme long» dans une chambre du motel, ses déplacements dans la chambre, et son obsession grandissante d'une «explosion» qui se prépare, et qui se révèle peu à peu surtout comme (mais pas seulement) une explosion dans sa tête. Se déroulant synchroniquement, mais parallèlement, jusqu'à leur entrecroisement violent à la fin du roman de Laure Angstelle, les histoires de lhomme long et de Mélanie correspondent à deux trajets narratifs plus généralisés qu'on pourrait appeler «son histoire à Lui» et «son histoire à Elle». Son histoire à Lui: celle de l'homme du vingtième siècle, piégé par les chiffres et les équations d'une science qui fut à l'origine quête de beauté, un homme dégoûté par son propre corps, et habité par la vision apocalyptique de la fin d'une culture érigée contre le féminin: I I am become / Death - maintenant nous sommes tous des fils de chiennes (p. 17). Son histoire à Elle: celle d'une jeune femme au seuil de la découverte de son désir, assoiffée et vulnérable, cherchant à exister pleinement et à «faire sens» de l'existence malgré le vide dont elle a hérité:

Très jeune, il n'y eut point d'avenir et le monde ressembla d̀ une maison incendiée comme celle qui le fut au coin de la rue par des hommes «étrangers» (p. 181).

J'avais quinze ans et de toutes mes forces j'appuyais sur mes pensées pour qu'elles penchent la réalité du côté de la lumière (p. 184).

L'interdépendance et l'affrontement inévitable de ces deux trajets narratifs dans la scène finale du meurtre d'Angela Parkins par l'homme long figurent l'énigme que tout le roman de Brossard explore et tente de résoudre 2 . Meurtre aussi froid, abstrait et dévastateur que l'explosion silencieuse par laquelle la technologie pourrait annihiler la terre, et que l'homme long semble avoir accompli par le seul pouvoir de son regard haineux. Meurtre qui survient au moment où Mélanie et Angela sont au seuil d'une rencontre - sensuelle, émotive et d'intelligence - qui aurait porté tout le sens du roman vers la possibilité d'une transformation culturelle:

Je ne connais pas vraiment Angela Parkins et voici pourtant nos corps rapprochés un instant, puis distants, lents et longs dans la distance de l'Amérique. Nous sommes inséparables et distantes en pleine éternité. Nous sommes le désert et l'évidence. Peut-être la nuit et la couleur de l'aube. Les femmes se sont rapprochées de nous... Elle parle, parle, part vers je ne sais où, elle dit que ça recommence parole, sentiers, papillons et qu'elle aime ça la lenteur obligée des mots... elle dit qu'il ne faut pas renoncer, que rien n'est impossible si la mémoire accomplit dans 
l'improbable la certitude qui en soi veille à l'horizon a la beauté, elle parle de l'attachement que nous avons pour certains mots et que ceux-ci sont comme de petites morts lentes dans la réalité consciente (p. 49-50).

Mais il faudrait citer tout le roman. Car c'est dans ses mots que réside la beauté qui transcende le désespoir de cette intrigue, mots qu'il faut goûter et garder en soi comme un vin exquis sur le palais, ou comme l'aube qu'on ne voit même pas quand on laisse d la nuit le soin de s'avancer dans nos vies d ce point que la lueur du jour s'effite dans nos yeux comme un lendemain frileux, [quand] dans le taxi blafard qui nous ramène d la maison... il n'y a plus de contexte et que nos sens broient du vide appelant le sommeil, d'autres artifices pour enrober le corps. Car l'aube se mérite, et, lorsqu'on a su la mériter, elle est au bout de l'âme une certitude tranquille, un apaisement des yeux épris de changements et d'utopies (p. 151).

1 Montréal, l'Hexagone, 1987.

2 Ressemblance structurale frappante avec les Fous de Bassan d'Anne Hébert, où les histoires paralleles de Stevens Brown et des deux jeunes filles qu'il finit par violer et assassiner représentent une autre version de l'entrecroisement tragique de «son histoire à Lui» et de «son histoire à Elle». 